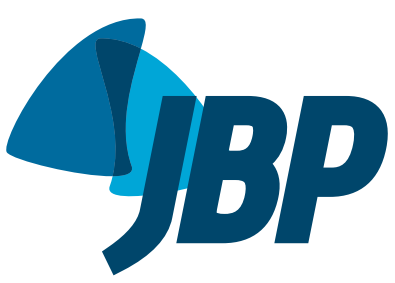

\section{Rapidly growing pulmonary ground-glass nodule caused by metastatic melanoma lacking uptake on ${ }^{18} \mathrm{~F}-\mathrm{FDG}$ PET-CT}

\author{
Giorgia Dalpiaz ${ }^{1}$ Sofia Asioli², Stefano Fanti ${ }^{3}$, Gaetano Rea ${ }^{4}$, Edson Marchiori ${ }^{5, a}$
}

\section{TO THE EDITOR:}

In November of 2003, a 33-year-old man underwent surgical treatment for a malignant melanoma on the chest. The mitotic rate was 4 mitoses $/ \mathrm{mm}^{2}$, and tumor thickness was $1.35 \mathrm{~mm}$, with no skin ulceration, lymphovascular invasion, or perineural invasion. After 5 years of follow-up, a CT scan showed a 15-mm subsolid nodule (pure ground-glass nodule) in the right lower lobe (Figure 1A). A follow-up CT scan performed 6 months later showed that the nodule had increased in size and had an eccentric solid component (Figure 1B). An ${ }^{18} \mathrm{~F}$-fluorodeoxyglucose positron emission tomography/ CT (FDG-PET/CT) scan showed that the nodule lacked FDG uptake (Figure 1C). Nodal and distant metastases were absent. Surgical resection of the lung lesion was performed. Histological examination showed the spread of neoplastic melanoma cells along the alveolar walls, with lepidic growth (Figure 1D). No hemorrhage was detected around the lesion. Immunohistochemical analysis of S-100 protein showed that the neoplastic melanoma cells were in close proximity to cytokeratin 7-positive normal alveolar epithelium. Therefore, the patient was diagnosed with metastatic lung cancer from a primary cutaneous melanoma. He was started on chemotherapy with dacarbazine and cisplatin but showed no signs of improvement. He died a few months later as a result of disease progression.

Subsolid nodules are CT findings that can be classified as pure or partially solid ground-glass nodules. Subsolid pulmonary nodules have been reported in association with various lung diseases, including non-neoplastic diseases, primary neoplasms, and metastatic neoplasms. The CT patterns of pulmonary parenchymal involvement by malignant melanoma vary. Multiple solid nodules constitute the most common CT finding. Metastatic pulmonary melanoma appearing as a solitary ground-glass opacity nodule is very uncommon. ${ }^{(1-3)}$ Negative FDG uptake on FDG-PET/CT is expected, as it is for other lesions with lepidic growth, such as peripheral lung adenocarcinomas (and their precursors) and metastases arising from adenocarcinoma of the gastrointestinal tract. ${ }^{(4)}$ Subsolid nodules have various benign and malignant etiologies. When persistent, subsolid pulmonary nodules are very likely to represent part of the pathological spectrum of lung adenocarcinoma. ${ }^{(5)}$ Although imaging findings were not pathognomonic in our patient, the rapid growth within a short period of time raised the suspicion of metastatic disease. ${ }^{(1,5)}$ FDG-PET/CT has a major role in nodal staging for decisions pertaining to surgical resection; the use of limited surgical resection in patients with subsolid nodules but no documented nodal metastasis is under investigation. ${ }^{(4)}$ Surgical resection is the preferred method for the histological diagnosis of subsolid nodules. ${ }^{(5)}$ In conclusion, in patients with malignant melanoma, a solitary subsolid pulmonary nodule that shows rapid growth over a few months should raise the suspicion of metastasis, despite negative FDG uptake on FDG-PET/CT.

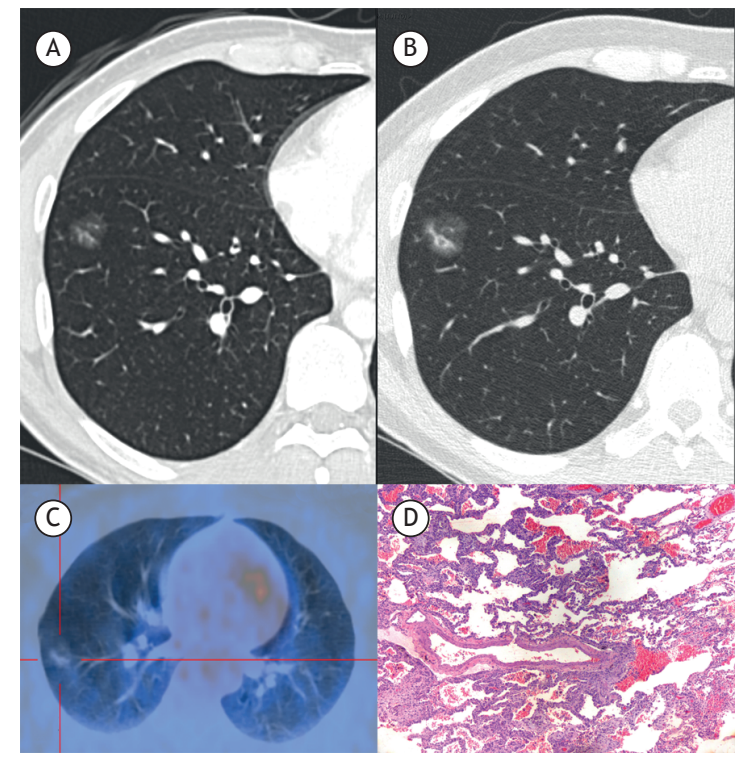

Figure 1. In A, axial CT scan of the chest showing a 15-mm subsolid nodule (pure ground-glass nodule) in the right lower lobe. In B, a CT scan of the chest taken 6 months after the first, showing that the nodule had increased in size and had an eccentric solid component. In C, ${ }^{18} \mathrm{~F}$-fluorodeoxyglucose positron emission tomography/CT scan showing that the nodule lacked ${ }^{18} \mathrm{~F}$-fluorodeoxyglucose uptake. In D, photomicrograph showing spread of neoplastic melanoma cells along the alveolar walls, with lepidic growth (H\&E staining; magnification, $\times 50$ ). Immunohistochemistry showed that the cells were positive for S-100 protein, which is a melanocytic marker (not shown). 


\section{REFERENCES}

1. Kang MJ, Kim MA, Park CM, Lee CH, Goo JM, Lee HJ. Ground-glass nodules found in two patients with malignant melanomas: different growth rate and different histology. Clin Imaging. 2010;34(5):396-9. https://doi.org/10.1016/j.clinimag.2009.10.036

2. Dalpiaz G, Kawamukai K, Parisi AM, La Torre L, Forcella D, Leuzz G. Ground-glass opacity of the lung in a patient with melanoma: "The radiological seed of doubt". Rev Esp Med Nucl Imagen Mol. 2015;34(6):390-2. https://doi.org/10.1016/j.remn.2015.04.006

3. Mizuuchi H, Suda K, Kitahara H, Shimamatsu S, Kohno M, Okamoto $T$, et al. Solitary pulmonary metastasis from malignant melanoma of the bulbar conjunctiva presenting as a pulmonary ground glass nodule: Report of a case. Thorac Cancer. 2015:6(1):97-100. https:// doi.org/10.1111/1759-7714.12124

4. Erasmus JJ, Macapinlac HA. Low-sensitivity FDG-PET studies: less common lung neoplasms. Semin Nucl Med. 2012;42(4):255-60. https://doi.org/10.1053/j.semnuclmed.2012.03.001

5. Naidich DP, Bankier AA, MacMahon H, Schaefer-Prokop CM Pistolesi M, Goo JM, et al. Recommendations for the management of subsolid pulmonary nodules detected at CT: a statement from the Fleischner Society. Radiology. 2015;266(1):304-17. https://doi. org/10.1148/radiol.12120628 